\title{
A mathematical expression for depth-light curves of therapeutic proton beams in a quenching scintillator
}

\author{
Laurent Kelleter ${ }^{1, *}$ and Simon Jolly ${ }^{1}$ \\ ${ }^{1}$ Dept. of Physics and Astronomy, University College London, Gower Street, WC1E 6BT London, UK
}

Purpose: Recently, there has been increasing interest in the development of scintillator-based detectors for the measurement of depth-dose curves of therapeutic proton beams ${ }^{1}$. These detectors allow the measurement of single beam parameters such as the proton range or the reconstruction of the full three-dimensional dose distribution. Thus, scintillation detectors could play an important role in beam quality assurance, on-line beam monitoring, and proton imaging. However, the light output of the scintillator as a function of dose deposition is subject to quenching effects due to the high specific energy loss of incident protons, particularly in the Bragg peak. The aim of this work is to develop a model that describes the percent depth-light (PDL) curve in a quenching scintillator and allow the extraction of information about the beam range and the strength of the quenching.

Methods: A mathematical expression of a depth-light curve, derived from a combination of Birks' law ${ }^{2}$ and Bortfeld's Bragg curve ${ }^{3}$ that is termed a "quenched Bragg" curve, is presented. The model is validated against simulation and measurement.

Results: A fit of the quenched Bragg model to simulated depth-light curves in a polystyrenebased scintillator shows good agreement between the two, with a maximum deviation of $2.5 \%$ at the Bragg peak. The differences are larger behind the Bragg peak and in the dose build-up region. In the same simulation, the difference between the reconstructed range and the reference proton range is found to be always smaller than $0.16 \mathrm{~mm}$. The comparison with measured data shows that the fitted beam range agrees with the reference range within their respective uncertainties.

Conclusions: The quenched Bragg model is, therefore, an accurate tool for the range measurement from quenched depth-dose curves. Moreover, it allows the reconstruction of the beam energy spread, the particle fluence and the magnitude of the quenching effect from a measured depth-light curve.

Keywords: proton therapy, Bragg curve, light quenching, Birks' law, plastic scintillator, beam range, quality assurance, Bortfeld model, pencil beam

\section{INTRODUCTION}

The gold standard for the measurement of percent depth-dose (PDD) curves in proton therapy are water phantoms with a movable ionisation chamber ${ }^{4}$. The accurate measurement of PDD curves plays an important role in beam quality assurance, online beam monitoring and imaging. However, scanning the water tank for a full measurement of a PDD curve is very time-consuming. Recently, there has been increasing interest in organic scintillator-based detectors for proton PDD curve measurements ${ }^{1}$. A scintillator is a material that produces light as a response to the energy deposition by ionising radiation. A percent depth-light (PDL) curve is defined as the scintillation light output versus penetration depth of the beam in the scintillator. Some advantages of organic scintillators over ionisation chambers are their low cost, high spatial resolution, the avoidance of any high voltage power supply, close-to waterequivalent density, dose-rate independence and scalability ${ }^{1}$.

To date, commercially available clinical applications for scintillators in proton therapy focus mainly on scintillating screens for beam spot measurements ${ }^{5}$. However, scintillation detectors that were originally developed for conventional X-ray radiotherapy have been successfully applied to proton depth-light curve measurements in scanning water phantoms $^{6}$. Similar to conventional water phantoms, the measurement of a full proton PDL curve with the scintillator can take several minutes.

Several groups therefore investigated the development of solely scintillator-based detectors (without water absorber) for fast proton PDL curve measurements at the speed of beam delivery, wherein large blocks of scintillator intercept the entire beam: prototype detectors of such kind were built using either plastic ${ }^{7,8}$ or liquid scintillator ${ }^{9}$. However, all of these detectors have in common that they need to correct for optical artefacts ${ }^{10}$ and quenching effects ${ }^{11}$.

In an organic scintillator, scintillation light is produced through the de-excitation of specific energy levels in organic molecules ${ }^{12}$. All alternative de-excitation modes that do not involve the production of a scintillation photon are

\footnotetext{
* laurent.kelleter@ucl.ac.uk
} 
grouped under the term "quenching". However, the percentage of dose converted to scintillation light depends on the local energy deposition density: this results in a non-linear response in the scintillation light output to absorbed dose.

In fact, the differential light output $d L / d z$ of an organic scintillator can be described by a nonlinear function of the differential energy loss $d E / d z$ (or Linear Energy Transfer, LET), first reported by Birks ${ }^{2}$. Birks suggested that the variable quenching is caused by temporarily "damaged" molecules along the track of the ionising particle. The density of the damaged molecules depends on the energy loss of the traversing particle. This relation is described by Birks' law (see equation 5). Others have suggested modifications to Birks' law ${ }^{13}$ or developed alternative models ${ }^{14}$. Recently, it was shown that Birks' constant is in fact not constant for low-energy X-rays ${ }^{15}$. Later, a different class of models emerged based on the Energy Deposition of Secondary Electrons (EDSE) ${ }^{16}$, which fixed the false prediction by Birks' model of a constant quenching factor for different ions with the same LET. EDSE models have been shown to be able to predict the PDL curve within $\pm 5 \% 17$.

However, it was found that Birks' law gives a good approximation of the light yield for energy losses up to $100 \mathrm{MeV} /\left(\mathrm{g} \mathrm{cm}^{-2}\right)^{18}$. The average energy loss of protons along the Bragg curve is found to be $<25 \mathrm{MeV} /\left(\mathrm{g} \mathrm{cm}^{-2}\right)$ in analytical calculations ${ }^{19}$ and simulations ${ }^{20}$. Moreover, Christensen et al. ${ }^{21}$ found that correction factors to Birks' model based on track structure theory are smaller than $2 \%$ for a proton LET below $45 \mathrm{MeV} / \mathrm{cm}$. In conclusion, the simplicity of Birks' model, which allows the quenching to be described with only one additional parameter, as well its satisfying accuracy, justify its use within the energy range utilised in proton therapy.

Robertson et al. ${ }^{11}$ used simulated three-dimensional LET distributions and Birks' model to correct for quenching effects in measured proton PDL curves. This correction was then used for fast measurements of the range of proton pencil beams ${ }^{22}$. It relies on the accurate matching of the predicted and the measured LET distributions and is therefore prone to small shifts between the simulated and the measured Bragg curve ${ }^{1,23}$. Range information has also been extracted from the scintillation light output without applying any correction for quenching at all ${ }^{9,24,25}$.

There exist commercially available multi-layer ionisation chambers (MLIC) for fast proton PDD curve measurements with a typical spatial resolution of $2 \mathrm{~mm}$ water-equivalent thickness. Those MLICs rely on an analytical approximation of the Bragg curve developed by Thomas Bortfeld ${ }^{3}$ for the accurate measurement (within 0.5 mm) of the proton range $^{26}$. The aim of this work is to make Bortfeld's formula applicable to scintillation detectors. A mathematical expression of a PDL curve in organic scintillators has been derived by combining Bortfeld's analytical approximation of a depth-dose curve with Birks' formula for light quenching. The combination of the two models is relatively straightforward because both models parametrise the LET distribution. Including quenching models based on trackstructure theory in Bortfeld's model would be more complex and require more additional parameters. The resulting "quenched Bragg" (QB) model enables the PDL curve to be modelled without prior knowledge of the LET distribution. In fact, the simultaneous fitting of the LET distribution and the quenching correction prevents the occurrence of alignment errors between the two, which is the main advantage of this approach. By fitting the quenched Bragg model to the depth-light curve one can reconstruct beam/material parameters such as proton range, beam current, initial energy distribution and Birks' constant.

\section{MATERIALS AND METHODS}

\section{A. Derivation of a quenched Bragg curve}

In this section the formula for a quenched Bragg curve is derived by combining Birks' formula for light quenching with Bortfeld's analytical approximation of a proton Bragg curve. The derivation follows closely the one performed by Thomas Bortfeld ${ }^{3}$ and is therefore less detailed. Prior knowledge of Bortfeld's original derivation is therefore recommended.

In order to derive an analytical approximation of a Bragg curve without quenching, Thomas Bortfeld began with the energy fluence $\Psi$ at depth $z$, being the product of the local particle fluence $\Phi(z)$ and the remaining mean proton energy $E(z)$. In an analogous way, the light fluence $\Theta(z)$ is considered, being the product of the local particle fluence $\Phi(z)$ and the remaining mean light output $L(z)$ of the beam. It is the light output $L(z)$ that takes into account the quenching relation between energy loss and scintillation light production.

$$
\Theta(z)=\Phi(z) L(z)
$$

The local scintillation light output per unit mass $U(z)$ can be obtained through differentiation of $\Theta(z)$ and normalisation to the material density $\rho$ :

$$
U(z)=-\frac{1}{\rho} \frac{d \Theta(z)}{d z}=-\frac{1}{\rho}\left(\Phi(z) \frac{d L(z)}{d z}+\frac{d \Phi(z)}{d z} L(z)\right)
$$


The proton fluence reduction represented by the factor $d \Phi / d z$ can be interpreted as being caused by non-elastic nuclear reactions that eliminate protons from the original beam. However, only a fraction of the energy going into these reactions is deposited locally and can therefore contribute to the production of scintillation light. As such, Bortfeld introduced a factor $\gamma$ which represents the fraction of locally absorbed energy from non-elastic nuclear interactions $^{3}$. This gives an expression for the depth-light curve $\hat{Q}(z)$.

$$
\hat{Q}(z)=-\frac{1}{\rho}\left(\Phi(z) \frac{d L(z)}{d z}+\gamma \frac{d \Phi(z)}{d z} L(z)\right)
$$

In its current form $\hat{Q}(z)$ does not account for beam range straggling. Bortfeld found that a good approximation is to fold $\hat{Q}(z)$ with a Gaussian with a constant standard deviation $\sigma$ in order to include the effect of range straggling. The initial depth dependence of $\sigma(z)$ can be neglected due to the relative flatness of the Bragg curve's plateau upstream of the Bragg peak ${ }^{3}$. The minimum value of $\sigma$ is determined by the range straggling of a mono-energetic beam $\sigma_{\text {mono }}$. In addition to that, $\sigma$ will further increase if the proton beam has an initial proton energy distribution with a non-zero width. Folding a Gaussian with standard deviation $\sigma$ into $\hat{Q}(z)$ yields a mathematical expression of a quenched Bragg curve $Q(z)$.

$$
Q(z)=\langle\hat{Q}\rangle(z)=\int_{0}^{R_{0}} \hat{Q}\left(z^{\prime}\right) \frac{1}{\sqrt{2 \pi} \sigma} \exp \left(-\left(z-z^{\prime}\right)^{2} / 2 \sigma^{2}\right) d z^{\prime}
$$

In order to calculate the depth-light curve, expressions need to be found for the light output and the particle fluence. First, the quenched light output of a scintillator is considered. According to Birks' law, the light yield $d L$ in a path segment $d z$ is a function of the differential energy loss $d E / d z$. Just like $d E / d z, d L / d z$ is a negative function as it describes the release of light or a decrease of the potential to emit light. Since Birks' constant is usually defined as being positive, the absolute value of the differential energy loss must be used, introducing a negative into the definition of $d L / d z$. Alternatively, Birks' constant could be defined as being negative.

$$
\frac{d L}{d z}=-\frac{S}{\left|\frac{d E}{d z}\right|^{-1}+k B}
$$

Here, $S$ is the scintillation light constant of the material and $k B$ is Birks' constant. Integration of $d L / d z$ from $z$ to the proton range $R_{0}$, which is defined as the mean stopping depth of the beam protons, gives the remaining light output $L$ at depth $z$. The remaining light output is positive, just like the remaining energy $E(z)$ of a proton.

$$
L(z)=-\int_{z}^{R_{0}} \frac{d L}{d z^{\prime}} d z^{\prime}
$$

In order to find an expression for the energy loss in the medium, Bortfeld's derivation is again used ${ }^{3}$. At the heart of Bortfeld's Bragg curve is the relation between the proton range $R_{0}$ and the initial beam energy $E_{0}$, approximated by a power law. The proton range $R_{0}$ coincides with the $80 \%$ fall-off of the depth-dose curve if nuclear reactions and the initial beam energy spread are neglected ${ }^{3}$. The parameters $\alpha$ and $p$ are material-specific constants that need to be determined from a fit of the power law to experimental or simulated data. The range-energy relationship, also known as Geiger's rule ${ }^{3}$, is given by:

$$
R_{0}=\alpha E_{0}^{p}
$$

The values for $\alpha$ and $p$ found for polystyrene in a Geant4-10-02 $27-29$ simulation (see Section IIB) differ slightly from those values found by Bortfeld for water (see table I). The uncertainties on $\alpha$ and $p$ are determined by varying the fit range of the power law. The residual proton energy $E$ at depth $z$ can be written as:

$$
E(z)=\alpha^{-1 / p}\left(R_{0}-z\right)^{1 / p}
$$

Differentiation yields the differential energy loss:

$$
\frac{d E(z)}{d z}=-\frac{1}{p \alpha^{1 / p}}\left(R_{0}-z\right)^{1 / p-1}
$$


TABLE I. Summary of variables and constants used in equation 13. Adapted from table 1 in $^{3}$. The given values are specific to polystyrene-based scintillator.

\begin{tabular}{llll}
\hline Variable & Description & Value & Unit \\
\hline$p$ & Exponent of range-energy relation & $1.753 \pm 0.010$ & 1 \\
$\alpha$ & Proportionality factor & $0.0237 \pm 0.0005$ & $\mathrm{~mm} / \mathrm{MeV}^{\mathrm{p}}$ \\
$\beta$ & Slope parameter of fluence reduction & $0.0012^{3}$ & $\mathrm{~mm}^{-1}$ \\
$\gamma$ & Fraction of locally absorbed energy & $0.6^{3}$ & 1 \\
$S$ & Scintillation light constant & $9,744^{30}$ & $\mathrm{photons} / \mathrm{MeV}$ \\
$\rho$ & Density of the scintillator & $1.03^{30}$ & $\mathrm{~g} / \mathrm{cm}^{3}$ \\
$k B$ & Birks' constant & fit parameter & $\mathrm{mm} / \mathrm{MeV}$ \\
$R_{0}$ & Proton range & fit parameter & $\mathrm{mm}$ \\
$\sigma$ & Width of Gaussian range straggling & fit parameter & $\mathrm{mm}$ \\
$\Phi_{0}$ & Fluence factor & fit parameter & $\mathrm{particles} / \mathrm{mm}^{2}$ \\
\hline
\end{tabular}

The range-energy relation also allows $\sigma$ to be decomposed into a component that takes into account the initial beam energy spread $\sigma_{E}$ and a mono-energetic component. Bortfeld found the relation:

$$
\sigma^{2}=\sigma_{\text {mono }}^{2}+\sigma_{E}^{2} \alpha^{2} p^{2} E_{0}^{2 p-2}
$$

where $\sigma_{E}$ is the Gaussian width of the initial proton beam energy spectrum and $\alpha$ and $p$ are parameters of Geiger's rule $^{3}$. For water, Bortfeld found that $\sigma_{\text {mono }}$ can be approximated by $\sigma_{\text {mono }}=0.012 R_{0}^{0.935}$.

The last undefined quantity in formula 4 is the proton fluence. Bortfeld approximated the normalised proton fluence $\Phi(z)$ as:

$$
\Phi(z)=\Phi_{0} \frac{1+\beta\left(R_{0}-z\right)}{1+\beta R_{0}}
$$

with $\beta$ being a slope parameter for the linear approximation of the fluence reduction due to non-elastic nuclear reactions and $\Phi_{0}$ being the initial proton fluence at depth $z=0$. Differentiation gives the change in proton fluence:

$$
\frac{d \Phi(z)}{d z}=-\Phi_{0} \frac{\beta}{1+\beta R_{0}}
$$

By inserting relations 3, 5, 6, 9,11 and 12 into equation 4 a mathematical expression of a quenched Bragg curve is obtained:

$$
\begin{aligned}
Q(z)= & \int_{0}^{R_{0}} \frac{1}{\rho}\left[\Phi_{0} \frac{1+\beta\left(R_{0}-z^{\prime}\right)}{1+\beta R_{0}} \frac{S}{\left(\frac{1}{p \alpha^{1 / p}}\left(R_{0}-z^{\prime}\right)^{1 / p-1}\right)^{-1}+k B}+\gamma \Phi_{0} \frac{\beta}{1+\beta R_{0}} \times\right. \\
& \left.\int_{z^{\prime}}^{R_{0}} \frac{S}{\left(\frac{1}{p \alpha^{1 / p}}\left(R_{0}-z^{\prime \prime}\right)^{1 / p-1}\right)^{-1}+k B} d z^{\prime \prime}\right] \frac{1}{\sqrt{2 \pi} \sigma} \exp \left(-\left(z-z^{\prime}\right)^{2} / 2 \sigma^{2}\right) d z^{\prime}
\end{aligned}
$$

For $k B=0$ and $S=1$, equation 13 transforms into Bortfeld's model. The integration over $z^{\prime}$ and $z^{\prime \prime}$ can be carried out numerically. In this work, the quenched Brag curve is implemented using the software toolkit ROOT ${ }^{31}$. The occurring integrals are solved using the function TF1: : Integral() with a relative precision of epsrel $=10^{-7}$ which is a compromise between computational speed and accuracy.

Bortfeld introduced an additional parameter $\epsilon$ in order to describe the deviation of the initial beam energy spectrum from a Gaussian shape. $\epsilon$ represents the size of a linear tail in the energy spectrum relative to $\Phi_{0}$. However, Bortfeld acknowledged that this is a coarse approximation which, while allowing Bortfeld's equation to be solved analytically, 
fails to describe realistic beam energy spectra ${ }^{3,32}$. As such, Bortfeld's energy-tail correction is not implemented in the quenched Bragg model. Further work is needed to develop the description of a realistic energy spectrum.

\section{B. Geant4 simulation}

The scintillation light output of a plastic scintillator irradiated by a proton beam is simulated using the Monte Carlo simulation toolkit Geant4-10-02 $2^{27-29}$. The implemented physics follows the recommendation of Hall et al. $(2015)^{33}$ : electromagnetic interactions are modelled using the G4EmStandardPhysics_option4. Nuclear interactions are modelled using G4HadronElasticPhyscis and G4HadronPhysicsQGSP_BIC_HP. In addition, G4EmExtraPhysics, G4StoppingPhysics, G4IonBinaryCascadePhysics and G4NeutronTrackingCut are included. The scintillation photons are simulated using G4Scintillation. G4EmSaturation is used to describe quenching effects with Birks' law. A step size of $0.1 \mathrm{~mm}$ and a range cut of $0.2 \mathrm{~mm}$ are used to guarantee high-resolution particle tracking.

Monoenergetic proton beams of $10^{6}$ particles with an initial transverse beam radius of $0 \mathrm{~mm}$ are used for all simulations. The initial beam energy spread and angular divergence are neglected. A $10 \times 10 \times 40 \mathrm{~cm}^{3}$ polystyrene $\left(C_{8} H_{8}\right)_{n}$ block is modelled with a density of $1.03 \mathrm{~g} / \mathrm{cm}^{330}$, a mean excitation energy of $I=68.7 \mathrm{eV}^{34}$ and a Birks' constant of $0.090 \mathrm{~mm} / \mathrm{MeV}^{23,35}$. The depth-light curve is obtained by recording the production depth $z$ of each optical photon produced in the scintillator. The resulting depth-light curve is obtained by filling the photon production depth $z$ into a histogram with $0.1 \mathrm{~mm}$ bin width.

\section{Experimental data}

The depth-light curves of proton beams with beam energies of $59.15 \mathrm{MeV}\left(R_{0, \text { ref }}=27.34 \pm 0.20 \mathrm{~mm}^{36}\right)$ and $120.92 \mathrm{MeV}\left(R_{0, \text { ref }}=105.48 \pm 0.20 \mathrm{~mm}^{36}\right)$ were measured using a scintillation detector at the Heidelberger IonenstrahlTherapiezentrum (HIT, Heidelberg, Germany). The detector was made of a polystyrene-based plastic scintillator segmented into 2-3 mm thick sheets. The scintillation light was read out by a large-scale CMOS sensor attached to the side of the sheet stack. The lateral size of each scintillator sheet was $10 \times 10 \mathrm{~cm}^{2}$. The total thickness of the scintillator stack was $12.4 \mathrm{~cm}$ which translates into a maximum observable beam energy of $\sim 125 \mathrm{MeV}$. Birks' constant of this scintillator is unknown; however, Birks' constant for polystyrene-based scintillators has been measured in the past: $k B=0.0094 \mathrm{~g} \mathrm{MeV}^{-1} \mathrm{~cm}^{-223}$ and $k B=0.009 \mathrm{~g} \mathrm{MeV}^{-1} \mathrm{~cm}^{-235}$. A detailed publication on the detector concept and characterisation is in preparation. The QB curve was subsequently fitted to the data in order to independently validate the model. The detector resolution is taken into account in the fit by integrating the light output predicted by the QB curve in each respective scintillator sheet.

\section{RESULTS AND DISCUSSION}

\section{A. Comparison with Bortfeld's Bragg curve}

In this section, the effect caused by the introduction of the quenching into Bortfeld's formula is analysed. Figure 1 shows a comparison of Bortfeld's analytical approximation of a Bragg curve with the quenched Bragg model for three typical clinical proton beam ranges. This comparison demonstrates the influence of introducing quenching effects. Within each plot both curves utilise the same parameters except for $k B=0.090 \mathrm{~mm} / \mathrm{MeV}^{23,35}$ and $S=9744^{30}$ which are specific to the QB model. Their values can be found in table I. The width of the range straggling $\sigma$ is set to be equal to $0.012 R_{0}^{0.9353}$ as suggested by Bortfeld which represents a mono-energetic proton beam with zero initial energy spread in water. The relative normalisation is chosen such that the values of the curves at depth $z=0$ are identical and the absolute normalisation is such that the maximum of Bortfeld's depth-dose curve is equal to one.

It can be seen that the introduction of quenching decreases the peak-to-plateau ratio of the Bragg curve. For short beam ranges, the maximum of the QB curve is around $60 \%$ of the corresponding Bragg curve. However, this difference decreases with increasing beam range: this is due to increased range straggling at higher beam energies which in turn increases the mean proton energy at the Bragg peak. A higher mean proton energy translates into a lower differential energy loss which means weaker quenching. 


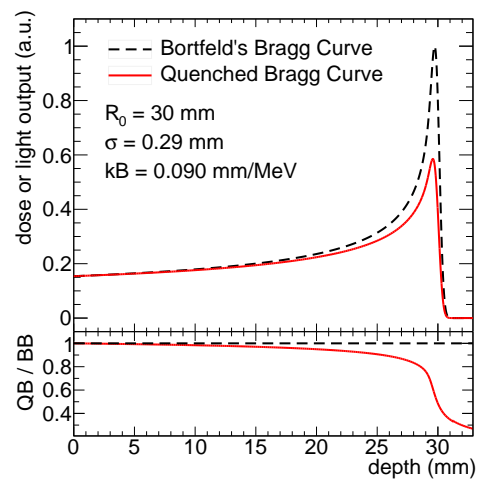

(a) $R_{0}=30 \mathrm{~mm}$

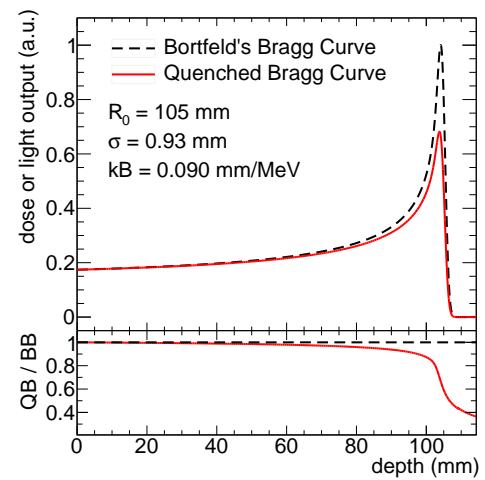

(b) $R_{0}=105 \mathrm{~mm}$

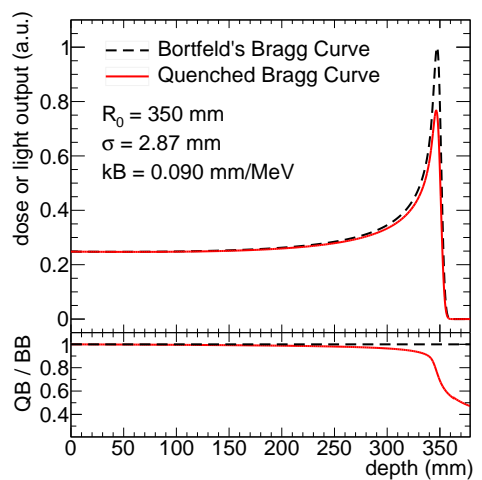

(c) $R_{0}=350 \mathrm{~mm}$

FIG. 1. Comparison of Bortfeld's Bragg curve with the quenched Bragg model of the same range for three typical clinical proton beam ranges. The effect of the introduced quenching is clearly visible around the Bragg peak.

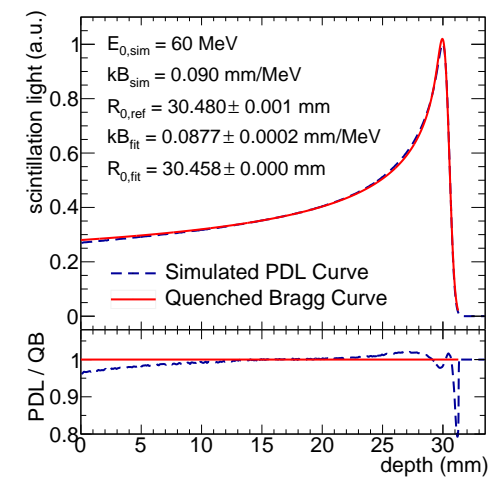

(a) $E_{0}=60 \mathrm{MeV}$

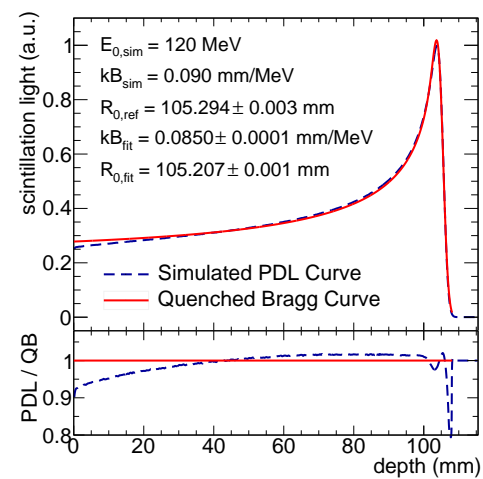

(b) $E_{0}=120 \mathrm{MeV}$

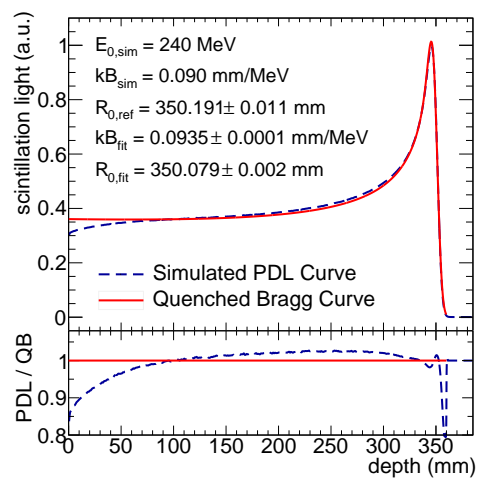

(c) $E_{0}=240 \mathrm{MeV}$

FIG. 2. Fit of the quenched Bragg model to a Geant4 simulation of a depth-light curve in a scintillator for three typical proton beam energies.

\section{B. Comparison with a simulated depth-light curve}

\section{General assessment}

In order to make a first assessment of the derived quenched Bragg model, a least-square fit of equation 13 to simulated depth-light curves is performed using the software toolkit ROOT. This comparison, however, has a limited meaningfulness because both the Geant4 simulation and the QB model utilise Birks' model to describe the quenching. The four fit parameters are the range $R_{0}$, the range straggling $\sigma$, the fluence $\Phi_{0}$ and Birks' constant $k B$. All remaining parameters are fixed. The fit range is from zero depth to a depth of $1.03 \times R_{0}$ because the $\mathrm{QB}$ model does not take into account the dose from secondary neutral particles which are responsible for the majority of the dose deposited behind the Bragg peak ${ }^{37}$. Figure 2 shows the fit for three typical clinical proton beam energies. The energy $E_{0, \text { sim }}$ in the plots corresponds to the simulated proton beam energy. Figure 2(a) shows a good agreement of the QB model with the simulation at $60 \mathrm{MeV}$ : discrepancies between the model and the simulation are below $2.5 \%$ at the Bragg peak. The difference is larger in the entrance region and behind the Bragg peak, where the light output falls below $20 \%$ of the value at the Bragg peak. At higher beam energies, the difference at the Bragg peak decreases to below $2 \%$ while the difference increases in the entrance region to a maximum of $15 \%$.

The observed discrepancies are of the same kind as reported by Bortfeld ${ }^{3}$ and can be explained by the coarse model of nuclear interactions in Bortfeld's analytical approximation (see also Bortfeld fit in figure 3) which is also present in the QB model. Dose build-up of secondary protons is responsible for the disagreement between the QB model and the simulation at the entrance to the medium ${ }^{37,38}$, which is particularly visible for high beam energies (figure 2 (c) for 


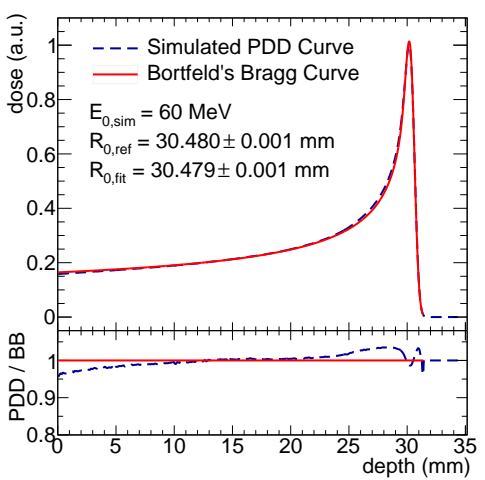

(a) $E_{0}=60 \mathrm{MeV}$

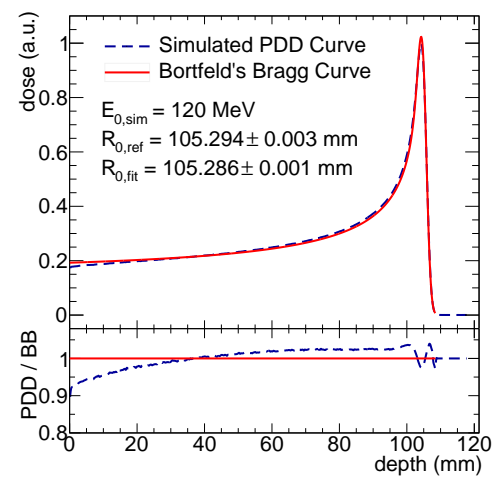

(b) $E_{0}=120 \mathrm{MeV}$

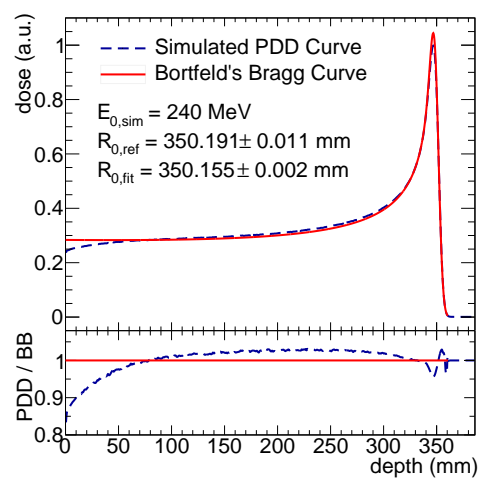

(c) $E_{0}=240 \mathrm{MeV}$

FIG. 3. Fit of Bortfeld's Bragg curve to a Geant4 simulation of a depth-dose curve in a scintillator for three typical proton beam energies.

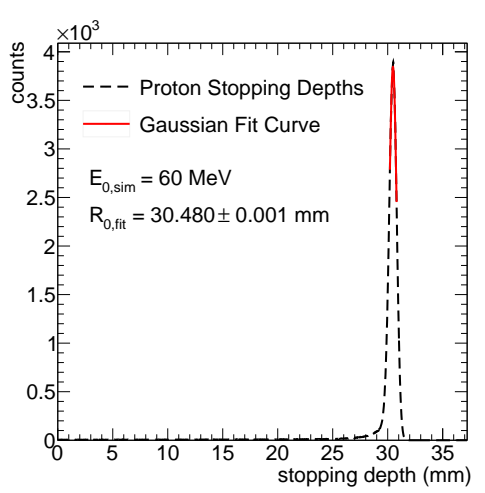

(a) $E_{0}=60 \mathrm{MeV}$

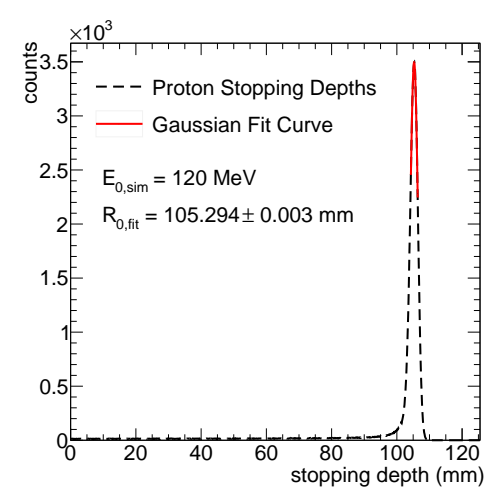

(b) $E_{0}=120 \mathrm{MeV}$

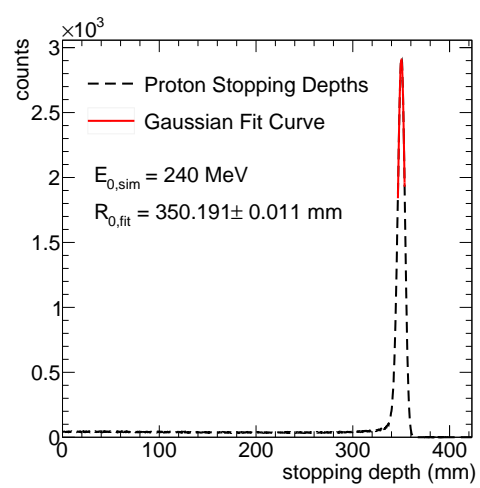

(c) $E_{0}=240 \mathrm{MeV}$

FIG. 4. Fit of a Gaussian curve to a histogram of the simulated proton stopping depths. The fit range is $\pm 1 \%$ around the expected proton range. The obtained range value is considered to be the best approximation of the true proton range and is henceforth used as a reference value.

$z<100 \mathrm{~mm}$ ). The difference upstream of the Bragg peak can also be explained by the coarse description of nuclear reactions and a possible overestimation of the peak width $\sigma$. Complex parameterisations of Bortfeld's Bragg curve have been proposed in the past in order to improve the matching between data and model ${ }^{39}$. These are not included in the QB model for the sake of simplicity but could be introduced in an analogous way as proposed by Zhang et al. ${ }^{39}$.

Since Bortfeld's analytical formula utilises the same parameters as the QB model, Bortfeld's formula can be used to reconstruct the initial depth-dose curve from the measured depth-light curve. This is done by calculating Bortfeld's depth-dose curve with the parameter values that are obtained from the fit of the QB model to the depth-light curve. However, the accuracy of the reconstructed depth-dose curve depends strongly on the accuracy of the fitted beam width $\sigma$ and is also subject to the model uncertainties discussed by Bortfeld ${ }^{3}$.

\section{Proton range reconstruction}

This work focuses on the range reconstruction performance of the QB model. In order to quantify the good agreement seen in figure 2 the proton beam ranges determined by three different methods are compared: the range from the QB model fit to the PDL (figure 2), the range from Bortfeld's Bragg curve fit to the PDD curve (figure 3) as well as the range from a Gaussian fit to the histogram of stopping depths of the simulated protons (figure 4).

The mean of the Gaussian fit to the histogram of proton stopping depths is defined as the reference proton range. However, it is non-trivial to obtain the true proton range from the histogram of the stopping depths. First, there 


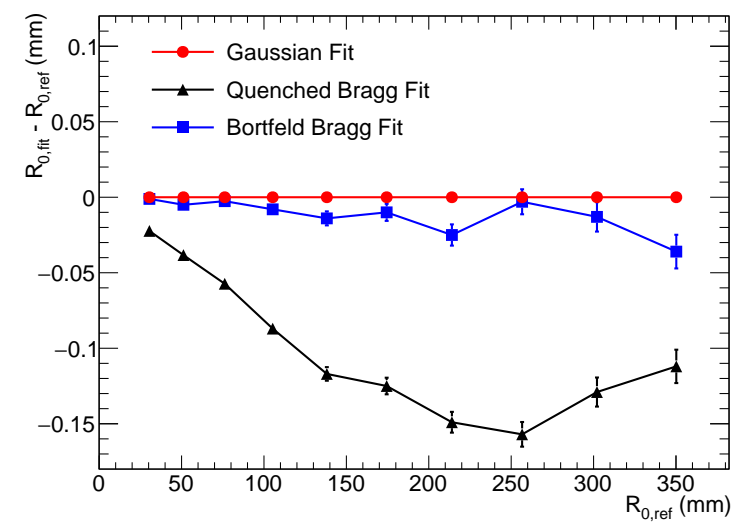

(a) Beam range

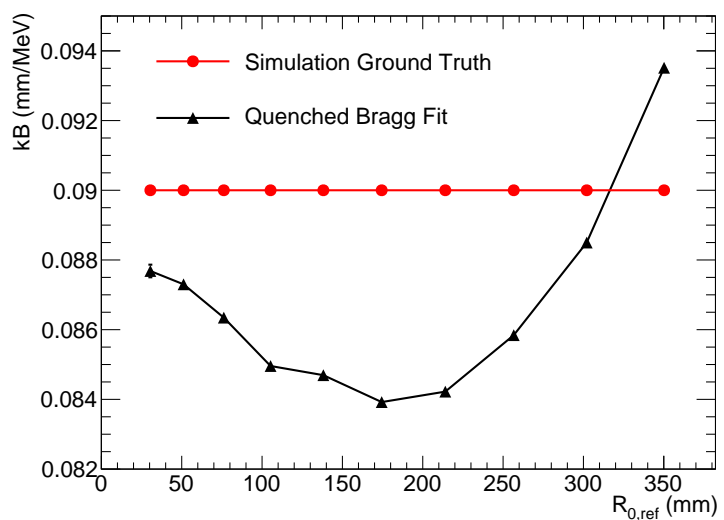

(b) Birks' constant

FIG. 5. Reconstruction of beam/material parameters from the simulated PDL curve using the quenched Bragg model for the typical range of clinical proton beam ranges.

are nuclear reactions removing protons from the beam and producing secondary particles. Second, the proton range is the projected path length of the protons which is always lower than the actual path length in a three-dimensional absorber. Those effects distort the initially Gaussian peak shape in the proton stopping depths histogram. In order to minimise the systematic uncertainty on the position of the peak maximum, the histogram is fitted in a narrow region of $\pm 1 \%$ around the expected proton range. The relative statistical uncertainty on the Gaussian fit is $0.003 \%$. The relative systematic difference between the true proton range (unknown ground truth) and the reference proton range (Gaussian fit) is estimated to be $<0.05 \%$.

Figure $5(\mathrm{a})$ shows the difference $\Delta R_{0}$ between each calculation method and the Gaussian fit, calculated for proton beam energies from 60 to $240 \mathrm{MeV}$ (corresponding to 30 to $350 \mathrm{~mm}$ range) in $20 \mathrm{MeV}$ steps. For the QB model fit, $\Delta R_{0}$ decreases with increasing beam range to a minimum of $-0.16 \mathrm{~mm}$ at $260 \mathrm{~mm}$ beam range. The maximum range difference for the Bortfeld fit is at a beam range of $350 \mathrm{~mm}$ and amounts to $0.04 \mathrm{~mm}$. The worse performance of the QB model fit in terms of range reconstruction can be explained by the additional parameter (Birks' constant) which opens up the parameter space for the range. The range reconstruction performance can be improved by fixing the value of Birks' constant, if known. Both the Bortfeld fit and the QB fit consistently underestimate the beam range, which is probably caused by the minimisation algorithm trying to compensate for model inaccuracies. The observed range difference can be interpreted as an estimate for the systematic model uncertainty on the range measurement.

The range measurement accuracy can be further improved by limiting the fitting range to a narrow region (e.g. $\pm 2 \%)$ around the expected proton range, thus avoiding the model uncertainties in the Bragg curve plateau. In this case, the maximum range difference is $0.11 \mathrm{~mm}$ for the Bortfeld fit and $0.025 \mathrm{~mm}$ for the QB model fit.

\section{Birks' constant reconstruction}

The quenched Bragg model can also be used to measure Birks' constant. Figure 5(b) shows the fitted value of Birks' constant in comparison with the ground truth in the simulation. Birks' constant is reconstructed within $\pm 7 \%$ for all simulated proton beams. The maximum deviation is found at a beam range of $175 \mathrm{~mm}$. There are large uncertainties associated with measurements of Birks' constants in plastic scintillators, which is also reflected in the large variation of values in the literature ${ }^{18,23,35,40}$. The QB model could help to reduce this uncertainty by providing a quick tool for measuring Birks' constant from PDL curves.

\section{Validation with experimental data}

The QB model is validated against experimental data. Figure 6 shows the measured depth-light curves of two different proton beam energies, together with the fitted QB model. The PDL curve is normalised to the peak light output. The values of the fit parameters and their uncertainties can be found in the figures. 


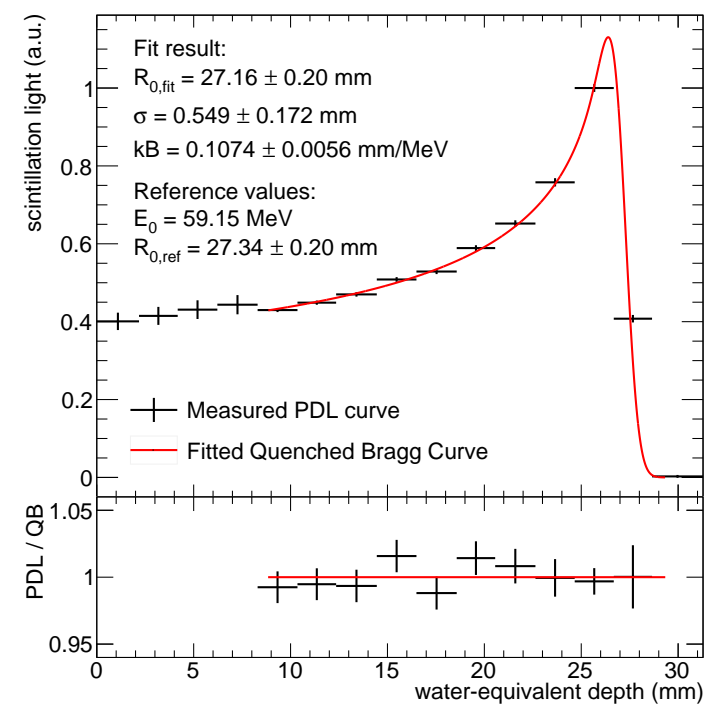

(a) $E_{0}=59.15 \mathrm{MeV}$

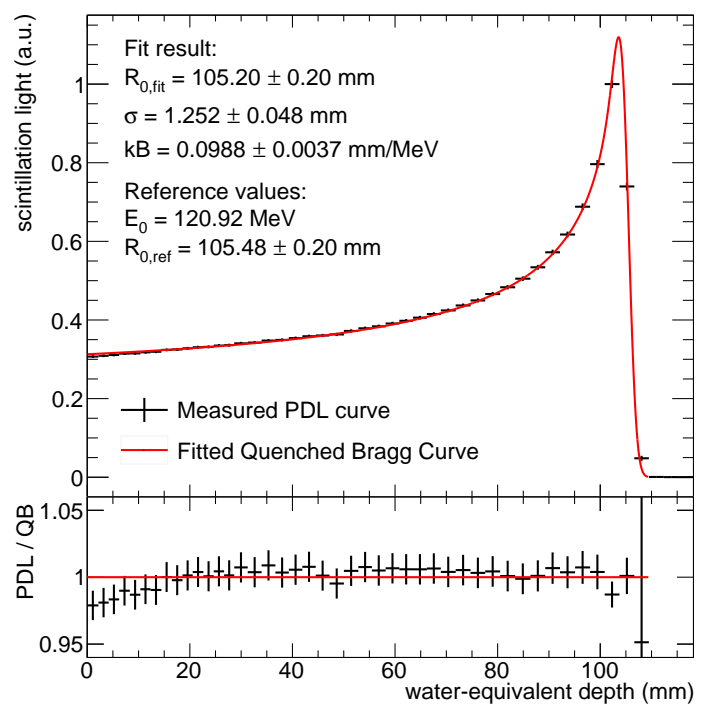

(b) $E_{0}=120.92 \mathrm{MeV}$

FIG. 6. Fit of the quenched Bragg model to measured PDL curves. The uncertainty on the fitted range $R_{0}$ only includes the uncertainty returned by the fitting process.

The fit range to the $59.15 \mathrm{MeV}$ proton beam is restricted to $9-30 \mathrm{~mm}$ because the large beam spot size at HIT resulted in protons hitting the CMOS sensor directly, resulting in a breakdown of the sensor calibration at depths below $9 \mathrm{~mm}$. The missing data points at the beam entrance probably lead to an overestimation of Birks' constant.

The measured beam ranges are $R_{0, \text { fit }}=27.16 \pm 0.20 \mathrm{~mm}$ and $R_{0, f i t}=105.20 \pm 0.20 \mathrm{~mm}$, which includes the uncertainty on the water-equivalent thickness of the scintillator. These values are consistent with the reference ranges within their uncertainties. The fitted values of Birks' constant are $k B=0.1074 \pm 0.0056 \mathrm{~mm} / \mathrm{MeV}(59.15 \mathrm{MeV})$ and $k B=0.0988 \pm 0.0037 \mathrm{~mm} / \mathrm{MeV}(120.92 \mathrm{MeV})$, which are similar to values reported in previous publications ${ }^{23,35}$.

The depth resolution of the detector is $2-3 \mathrm{~mm}$ (scintillator sheet thickness), which is too large to resolve the Bragg peak region to the same degree as in simulation. This also leads to an overestimation of the fitted beam width $\sigma$ and a larger uncertainty on the fitted Birks' constant. However, this also demonstrates the strength of the QB model since it allows some beam parameters to be reconstructed with a remarkable degree of accuracy, despite the coarse spatial resolution. Future work will be needed to validate the QB model against high-resolution PDL curves in order to resolve structures at the Bragg peak. The validation with higher-energy data above $120.92 \mathrm{MeV}$ is also subject of future work.

\section{Model extension to ion beams}

This work focuses on the description of PDL curves for protons. In order to expand the quenched Bragg model to ion beams, it is in principle sufficient to adapt the energy-range relation, i.e. the values of $\alpha$ and $p$. However, in addition, the dose contribution from projectile fragmentation must be considered. Instead of adapting the particle fluence $\Phi(z)$ in Bortfeld's model, the PDD model developed by Krämer et al. ${ }^{41}$ could be extended with a quenching correction. Krämer's Bragg curve uses extensive nuclear cross-section libraries to describe nuclear reactions between the projectile and the material and is therefore more complex to implement. A QB model based on Krämer's model would also benefit the modelling of proton PDL curves because of the more accurate description of the proton energy loss via nuclear reactions with the absorber.

\section{CONCLUSION}

The mathematical expression of a quenched depth-dose curve has been derived by combining Birks' law and Bortfeld's Bragg curve and compared with associated simulations of the scintillator light production and dose deposition. 
The strength of the approach is the simultaneous fitting of the quenching correction and the LET distribution which avoids misalignment uncertainties. The effect of quenching is demonstrated by a comparison of the resulting model of a quenched Bragg curve to Bortfeld's original Bragg curve. A fit of the quenched Bragg model to the simulated depth-light curve in a polystyrene-based scintillator shows that simulation and model agree within $2.5 \%$ in the Bragg peak. An analysis of the accuracy of the range reconstruction in simulation shows a maximum range difference of $0.16 \mathrm{~mm}$ between the range determined by a quenched Bragg model fit and the reference proton range. Birks' constant is reconstructed within $\pm 7 \%$. A comparison to measured data shows that the fitted range values agree with their reference values within their quoted sub-millimetre uncertainties. The quenched Bragg model is therefore believed to be an accurate tool for measuring the range of a proton beam from a depth-light curve, especially in the case of low depth resolution. Finally, since the quenched Bragg model and Bortfeld's Bragg curve utilise the same set of parameters, it is possible to use Bortfeld's formula to reconstruct the original depth-dose curve in a quenching medium from the fit parameters of the quenched Bragg model.

\section{ACKNOWLEDGMENTS}

This project has received funding from the European Union's Horizon 2020 research and innovation programme under the Marie Sklodowska-Curie grant agreement No 675265, OMA - Optimization of Medical Accelerators.

\section{CONFLICT OF INTEREST STATEMENT}

The authors have no relevant conflicts of interest to disclose.

\section{REFERENCES}

[1] Luc Beaulieu and Sam Beddar. Review of plastic and liquid scintillation dosimetry for photon, electron, and proton therapy. Phys. Med. Biol., 61, 2016.

[2] J B Birks. Scintillations from organic crystals: Specific fluorescence and relative response to different radiations. Proc. Phys. Soc. A, 64:874, 1951.

[3] Thomas Bortfeld. An analytical approximation of the bragg curve for therapeutic proton beams. Med. Phys., $24,1997$.

[4] Christian P Karger, Oliver Jaekel, Hugo Palmans, and Tatsuaki Kanai. Dosimetry for ion beam radiotherapy. Phys. Med. Biol., 55, 2010.

[5] S Russo, A Mirandola, S Molinelli, E Mastella, A Vai, G Magro, A Mairani, D Boi, M Donetti, and M Ciocca. Characterization of a commercial scintillation detector for 2-d dosimetry in scanned proton and carbon ion beams. Phys Med, 34, 2017.

[6] C Hoehr, C Lindsay, J Beaudry, C Penner, V Strgar, R Lee, and C Duzenli. Characterization of the exradin w1 plastic scintillation detector for small field applications in proton therapy. Phys. Med. Biol., 63, 2018.

[7] Mansour Almurayshid, Yusuf Helo, Andrzej Kacperek, Jennifer Griffiths, Jem Hebden, and Adam Gibson. Quality assurance in proton beam therapy using a plastic scintillator and a commercially available digital camera. Journal of applied cinical medical physics, 2017.

[8] D Mazzucconi, S Agosteo, M Ferrarini, L Fontana, V Lante, M Pullia, and S Savazzi. Mixed particle beam for simultaneous treatment and online range verification in carbon ion therapy: Proof-of-concept study. Med. Phys., $42,2018$.

[9] Chinmay D. Darne, Fahed Alsanea, Daniel G. Robertson, Narayan Sahoo, and Sam Beddar. Performance characterization of a 3d liquid scintillation detector for discrete spot scanning proton beam systems. Phys Med Biol., $62,2017$.

[10] Daniel Robertson, Cheukkai Hui, Louis Archambault, Radhe Mohan, and Sam Beddar. Optical artefact characterization and correction in volumetric scintillation dosimetry. Phys Med Biol., 59, 2014.

[11] Daniel Robertson, Dragan Mirkovic, Narayan Sahoo, and Sam Beddar. Quenching correction for volumetric scintillation dosimetry of proton beams. Phys Med Biol., 58, 2013.

[12] Glenn F. Knoll. Radiation Detection and Measurement. Wiley, 1979.

[13] C. N. Chou. The nature of the saturation effect of fluorescent scintillators. Phys. Rev., 87:904-905, Sep 1952.

[14] G. T. Wright. Scintillation response of organic phosphors. Phys. Rev., 91:1282-1283, Sep 1953.

[15] Jonathan Boivin, Sam Beddar, Chris Bonde, Daniel Schmidt, Wesley Culberson, Maxime Guillemette, and Luc Beaulieu. A systematic characterization of the low-energy photon response of plastic scintillation detectors. Physics in Medicine and Biology, 61(15):5569-5586, jul 2016. 
[16] K. Michaelian and A. Menchaca-Rocha. Model of ion-induced luminescence based on energy deposition by secondary electrons. Phys. Rev. B, 49:15550-15562, Jun 1994.

[17] F Alsanea and S Beddar. Evaluating analytical ionization quenching correction models for 3d liquid organic scintillator detector. Journal of Physics: Conference Series, 847:012022, may 2017.

[18] G D Badhwar, C L Deney, B R Dennis, and M F Kaplon. The non-linear response of the plastic scintillator NE102. Nucl. Instr. and Meth., 57, 1967.

[19] Jan J Wilkens and Uwe Oelfke. Analytical linear energy transfer calculations for proton therapy. Medical Physics, 30, 2003.

[20] Clemens Grassberger and Harald Paganetti. Elevated LET components in clinical proton beams. Phys. Med. Biol., 56, 2011.

[21] Jeppe Brage Christensen and Claus E Andersen. Relating ionization quenching in organic plastic scintillators to basic material properties by modelling excitation density transport and amorphous track structure during proton irradiation. Physics in Medicine \&s Biology, 63(19):195010, sep 2018.

[22] Cheuk Kai Hui, Daniel Robertson, Fahed Alsanea, and Sam Beddar. Fast range measurement of spot scanning proton beams using a volumetric liquid scintillator detector. Biomedical Physics 6 Engineering Express, 1(2):025204, jul 2015.

[23] L L W Wang, L A Perles, L Archambault, N Sahoo, D Mirkovic, and S Beddar. Determination of the quenching correction factors for plastic scintillation detectors in therapeutic high-energy proton beams. Physics in Medicine and Biology, 57(23):7767-7781, nov 2012.

[24] Thomas Henry, Daniel Robertson, Francois Therriault-Proulx, and Sam Beddar. Determination of the range and spreadout bragg peak width of proton beams using a large-volume liquid scintillator. International Journal of Particle Therapy., 2017.

[25] Yasuhiro Fukushima, Minoru Hamada, Teiji Nishio, and Koichi Maruyama. Development of an easy-to-handle range measurement tool using a plastic scintillator for proton beam therapy. Phys. Med. Biol., 51, 2006.

[26] Sandeep Dhanesar, Narayan Sahoo, Matthew Kerr, M. Brad Taylor, Paige Summers, X. Ronald Zhu, Falk Poenisch, and Michael Gillin. Quality assurance of proton beams using a multilayer ionization chamber system. Med. Phys., $40,2013$.

[27] S. Agostinelli et al. Geant4: A Simulation toolkit. Nucl. Instrum. Meth., A506:250-303, 2003.

[28] J. Allison et al. Geant4 developments and applications. IEEE Transactions on Nuclear Science, 53(1):270-278, Feb 2006.

[29] J. Allison et al. Recent developments in Geant4. Nuclear Instruments and Methods in Physics Research Section A: Accelerators, Spectrometers, Detectors and Associated Equipment, 835:186 - 225, 2016.

[30] NuviaTech Instruments. NuDET Plastic: Specification Sheet, October 2019.

[31] R. Brun and F. Rademakers. ROOT: An object oriented data analysis framework. Nucl. Instrum. Meth., A389:81-86, 1997.

[32] Thomas Tessonnier, Tiago Marcelos, Andrea Mairani, Stephan Brons, and Katia Parodi. Phase space generation for proton and carbon ion beams for external users and applications at the heidelberg ion therapy center. Frontiers in Oncology, $5: 297,2016$.

[33] David C Hall, Anastasia Makarova, Harald Paganetti, and Bernard Gottschalk. Validation of nuclear models in geant4 using the dose distribution of a $177 \mathrm{MeV}$ proton pencil beam. Physics in Medicine and Biology, 61(1):N1-N10, nov 2015.

[34] Berger M J, J S Coursey, M A Zucker, and J Chang. Stopping-power and range tables for electrons, protons, and helium ions. NIST Standard Reference Database 124, 2017.

[35] V.I. Tretyak. Semi-empirical calculation of quenching factors for ions in scintillators. Astroparticle Physics, 33(1):40 - 53, 2010.

[36] K K Parodi, A Mairani, S Brons, B G Hasch, F Sommerer, J Naumann, O Jaekel, T Haberer, and J Debus. Monte carlo simulations to support start-up and treatment planning of scanned proton and carbon ion therapy at a synchrotron-based facility. Phys Med Biol., 57, 2019.

[37] Laurent Kelleter, Benjamin Zhen Hong Tham, Ruben Saakyan, Jennifer Griffiths, Richard Amos, Simon Jolly, and Adam Gibson. Technical note: Simulation of dose buildup in proton pencil beams. Med. Phys., 2019.

[38] Wayne D Newhauser and Rui Zhang. The physics of proton therapy. Phys. Med. Biol., 60, 2015.

[39] Xiaodong Zhang, Wei Liu, Yupeng Li, Xiaoqiang Li, Michelle Quan, Radhe Mohan, Aman Anand, Narayan Sahoo, Michael Gillin, and Xiaorong R Zhu. Parameterization of multiple bragg curves for scanning proton beams using simultaneous fitting of multiple curves. Physics in Medicine and Biology, 56(24):7725-7735, nov 2011.

[40] L. Torrisi. Plastic scintillator investigations for relative dosimetry in proton-therapy. Nuclear Instruments and Methods in Physics Research Section B: Beam Interactions with Materials and Atoms, 170(3):523 - 530, 2000.

[41] M Kraemer, O Jaekel, T Haberer, G Kraft, D Schardt, and U Weber. Treatment planning for heavy-ion radiotherapy: physical beam model and dose optimization. Physics in Medicine and Biology, 45(11):3299-3317, oct 2000. 